\title{
LIMIT C*-ALGEBRAS ASSOCIATED WITH AN AUTOMORPHISM
}

\author{
BARUCH SOLEL*
}

\begin{abstract}
We present and study $\mathrm{C}^{*}$-algebras generated by "periodic weighted creation operators" on the Fock space associated with an automorphism $\alpha$ on a $C^{*}$-algebra $A$. These algebras can be viewed as generalized Bunce-Deddens algebras associated with the automorphism and can be written as a certain direct limit. We prove a crossed product presentation for such an algebra and find a necessary and sufficient condition for it to be simple. In the case where the automorphism is induced by an irrational rotation (on $C(\mathrm{~T})$ ) we compute the $\mathrm{K}$-theory groups and obtain a complete classification of these algebras.
\end{abstract}

\section{Introduction}

The purpose of this paper is to to present and study a class of $\mathrm{C}^{*}$-algebras generalizing the Bunce-Deddens algebras.

Recall that the Bunce-Deddens algebra $\mathscr{B}\left(\left\{n_{k}\right\}\right)$, associated with the sequence $\left\{n_{k}\right\}$, is a quotient (by the compact operators) of the $\mathrm{C}^{*}$-algebra generated by all weighted shifts (with respect to a fixed basis) of period $n_{k}$ for some $k \geq 1$ ([2]). In fact, for a fixed $k \geq 1$, the $\mathrm{C}^{*}$-algebra of all weighted shifts of period $n_{k}$ is isomorphic to the algebra of all $n_{k} \times n_{k}$ matrices over the Toeplitz algebra and its quotient by the compacts is isomorphic to $M_{n_{k}}(C(\mathrm{~T}))$. Thus the Bunce-Deddens algebra is a direct limit of the algebras $M_{n_{k}}(C(\mathrm{~T}))$.

A natural noncommutative generalization of the unilateral shift is obtained by considering creation operators on Fock Hilbert space. These operators were studied in applications to quantum physics and in free probability. More recently, creation operators, and the algebras that they generate, were studied in the context of Fock spaces of $C^{*}$-correspondences (also called Hilbert $C^{*}$ bimodules). This was initiated by M. Pimsner ([10]) and followed by several other authors (e.g. [6], [7], [4], [12]).

In a recent paper, D. Kribs introduced the concept of periodic weighted shifts on the full Fock space associated with a Hilbert space of dimension $N$ ([5]). The $\mathrm{C}^{*}$-algebra that he obtained (taking the appropriate quotient of the

\footnotetext{
* Supported by the Fund for the Promotion of Research at the Technion.

Received January 15, 2003.
} 
$\mathrm{C}^{*}$-algebra generated by all periodic weighted shifts of period $\left.n_{k}\right)$ is a direct limit of algebras of the form $M_{d_{k}}\left(O_{N^{k}}\right)$ where $d_{k}$ are certain integers and $O_{N^{k}}$ is the Cuntz algebra (with $N^{k}$ generators).

In the present paper we start with an automorphism $\alpha$ on a $\mathrm{C}^{*}$-algebra $A$ and form the (full) Fock space $\mathscr{F}$ associated with the pair $(A, \alpha)$ (i.e. associated with the bimodule ${ }_{\alpha} A$ ). As Pimsner showed in [10], the $\mathrm{C}^{*}$-algebra generated by the creation operators on this Fock space is the Toeplitz extension, $\mathscr{T}(A, \alpha)$, of the crossed product $A \times_{\alpha}$ Z. Letting $K(\mathscr{F})$ denote the compact operators on $\mathscr{F}$ (in the sense of compact operators on a $\mathrm{C}^{*}$-module), the crossed product is the quotient of $\mathscr{T}(A, \alpha)$ by $K(\mathscr{F})$. We shall define the concept of "periodic weighted creation operators" (more precisely, we define, in Definition 2.1, weighted representations of ${ }_{\alpha} A$ on $\mathscr{F}$ ) and consider the $\mathrm{C}^{*}$-algebra generated by all these operators with a fixed period $n$. This algebra turns out to be isomorphic to $M_{n}\left(\mathscr{T}\left(A, \alpha^{n}\right)\right)$ and, taking an appropriate quotient, we get $M_{n}\left(A \times_{\alpha^{n}} Z\right)$. Now let $\left\{n_{k}\right\}$ be an increasing sequence of positive integers with $n_{k} \mid n_{k+1}$. Considering the $\mathrm{C}^{*}$-algebra generated by all "periodic weighted creation operators" of period $n_{k}$ for some $k \geq 1$ and taking an appropriate quotient we get a certain direct limit of the algebras $M_{n_{k}}\left(A \times_{\alpha^{n_{k}}} Z\right)$. We shall write $\mathscr{B}_{\alpha}\left(\left\{n_{k}\right\}\right)$ for this $\mathrm{C}^{*}$-algebra. It can be thought of as a "generalized BunceDeddens algebra associated with $(A, \alpha)$ ". The details of this construction are presented in Section 2. We also show there that the algebra depends, up to an isomorphism, on the supernatural number of $\left\{n_{k}\right\}$ (and not on the sequence itself).

In Section 3 we study the structure of the algebra $\mathscr{B}_{\alpha}\left(\left\{n_{k}\right\}\right)$. We present necessary and sufficient conditions for it to have a unique tracial state (Theorem 3.1). In Theorem 3.4 we show that the algebra $\mathscr{B}_{\alpha}\left(\left\{n_{k}\right\}\right)$ can also be written as a crossed product $C(X, A) \times_{\sigma} \mathrm{Z}$. We then use it in Theorem 3.5 to prove a necessary and sufficient condition for simplicity of the algebra.

In Section 4 we specialize to the case where $A=C(\mathrm{~T})$ and $\alpha$ is an irrational rotation by $\theta$ (so that $A \times{ }_{\alpha} Z$ is the irrational rotation algebra $A_{\theta}$ ). In this case we use the notation $\mathscr{B}_{\theta}\left(\left\{n_{k}\right\}\right)$ for the algebra in order to emphasize the dependence on $\theta$. It follows from the previous section that the algebra is simple and has a unique tracial state $\tau$. We show that the $K_{0}$ and $K_{1}$ groups of the algebra are both isomorphic to $Q(\delta) \oplus \mathrm{Z}$ (where $\delta$ is the supernatural number of the sequence $\left\{n_{k}\right\}$ and $Q(\delta)$ is the group of rational numbers that can be written as a quotient $m / n_{k}$ for some $k$ ). We also find that the image of the map $\tau_{*}$, defined on the $K_{0}$ group, is $Q(\delta)+\theta \mathrm{Z}$. It then follows that two such algebras, with $\theta_{1}$, $\theta_{2}$ and supernatural numbers $\delta_{1}, \delta_{2}$ are isomorphic if and only if $\delta_{1}=\delta_{2}$ and either $\theta_{1}+\theta_{2}$ or $\theta_{1}-\theta_{2}$ belongs to $Q\left(\delta_{1}\right)$ (Corollary 4.2). 


\section{Preliminaries}

Let $A$ be a (unital) separable $\mathrm{C}^{*}$-algebra and let $\alpha$ be a unital automorphism of $A$. Write $E={ }_{\alpha} A$ for the $\mathrm{C}^{*}$-correspondence associated with this automorphism. As a vector space, $E$ is simply $A$. The bimodule structure is defined by

$$
a \cdot b \cdot c=\alpha(a) b c .
$$

Here $a, c$ are in $A$ and $b$ is in $A$ viewed as an element of $E$. The inner product is

$$
\left\langle b_{1}, b_{2}\right\rangle=b_{1}^{*} b_{2} .
$$

This makes $E$ into a $\mathrm{C}^{*}$-correspondence in the sense of [6]. It is easy to check that $E^{\otimes k}={ }_{\alpha^{k}} A$ and the isomorphism of $E^{\otimes k} \otimes E^{\otimes m}$ onto $E^{\otimes(k+m)}$ is given by

$$
V_{k, m}(a \otimes b)=\alpha^{m}(a) b
$$

for $a \in_{\alpha^{k}} A$ and $b \in_{\alpha^{m}} A$. In what follows we tend to suppress $V_{k, m}$ and identify the two spaces.

The Fock space associated with ${ }_{\alpha} A=E$ is

$$
\mathscr{F}(E)=A \oplus E \oplus E^{\otimes 2} \oplus \cdots .
$$

As a $\mathrm{C}^{*}$-module it is just the direct sum of infinitely many copies of $A$. To distinguish between elements of $\alpha_{\alpha^{k}} A$ for different $k$ 's, we write $\delta_{k}$ for the unit of $A$ viewed as an element of $\alpha_{\alpha^{k}} A$. Thus $\delta_{k} a$ would be $a$ as an element of $\alpha_{\alpha^{k}} A$. The Fock space has a left action that makes it into a $\mathrm{C}^{*}$-correspondence:

$$
\phi_{\infty}(a) \delta_{k} b=\delta_{k} \alpha^{k}(a) b .
$$

We shall denote the Fock space associated with $\alpha^{k}$ by $\mathscr{F}^{(k)}$ and the left action there by $\phi_{\infty}^{(k)}$.

Every $a$ in $E$ defines a shift operator on $\mathscr{F}(=\mathscr{F}(E))$ by

$$
T(a) \delta_{k} b=\delta_{1} a \otimes \delta_{k} b=\delta_{k+1} \alpha^{k}(a) b .
$$

(In the last equality we omitted the reference to $V_{1, k}$.)

A similar operator on $\mathscr{F}^{(k)}$ will be denoted $T^{(k)}(a)$.

Definition 2.1. Let $\lambda=\left\{\lambda_{i}: 1 \leq i<\infty\right\}$ be a bounded sequence of positive elements of $A$. The weighted representation of $E$ on $\mathscr{F}$ is

$$
T_{\lambda}(a) \delta_{k} b=\delta_{k+1} \alpha^{k}\left(\lambda_{k+1} a\right) b
$$

for $a, b \in A$. The weighted representation will be said to be periodic of period $k$ if for all $i \geq 1, \lambda_{i+k}=\lambda_{i}$. 
Note. The word "representation" above refers only to the fact that it is a representation of $E$ as a right module over $A$ (i.e. $T_{\lambda}(a \cdot c)=T_{\lambda}(a) \phi_{\infty}(c)$ for $c \in A$ and $a \in E$ ).

Note that every operator on $\mathscr{F}$ can be written as an infinite matrix (with respect to the decomposition of $\mathscr{F}$ as an infinite direct sum). It will be convenient to denote by $a \delta_{i j}$ the operator that maps $\delta_{j} b$ to $\delta_{i} a b$ (for $a$ in $A$ ).

Also we shall write $S$ for $T$ (1) (hence $S \delta_{k} a=\delta_{k+1} a$ ).

Definition 2.2. The $C^{*}$-algebra generated by the operators $\left\{\phi_{\infty}^{(k)}(A)\right.$, $\left.T^{(k)}\left(\alpha^{k} A\right)\right\}$ on $\mathscr{F}^{(k)}$ is called the Toeplitz algebra associated with $\alpha^{k}$ and will be denoted $\mathscr{T}_{k}$. Since $\mathscr{F}^{(k)}$ is a subspace of $\mathscr{F}$, each $\mathscr{T}_{k}$ can be viewed as a subspace of $\mathscr{L}(\mathscr{F})$.

Lemma 2.3. Given $k \geq 1$, the $C^{*}$-algebra generated by all the periodic representations of period $k$ (on $\mathscr{F}$ ) is isomorphic to the algebra of all $k \times k$ matrices over $\mathscr{T}_{k}$.

Proof. We start by setting some notation. We can write

$$
\mathscr{F}=\mathscr{F}_{k} \oplus\left(E \otimes \mathscr{F}_{k}\right) \oplus\left(E^{\otimes 2} \otimes \mathscr{F}_{k}\right) \oplus \cdots \oplus\left(E^{\otimes(k-1)} \otimes \mathscr{F}_{k}\right)
$$

Let us write $\mathcal{M}_{l}$ for $E^{\otimes l} \otimes \mathscr{F}_{k}, 0 \leq l \leq k-1$. Then $\mathscr{F}=\mathscr{M}_{0} \oplus \mathscr{M}_{1} \oplus \cdots \oplus \mathscr{M}_{k-1}$. Every operator on $\mathscr{F}$ can be written in a matricial way with respect to this decomposition. We shall write $X e_{i j}$ for the operator whose $i, j$ entry is $X$ ( $X$ maps $\mathcal{M}_{j}$ into $\left.\mathcal{M}_{i}\right)$ and all other entries vanish.

Note that, for all $0 \leq l \leq k-1, \phi_{\infty}(A) \mathscr{M}_{l} \subseteq \mathcal{M}_{l}$ so that the matrix of $\phi_{\infty}(a)$ for $a \in A$ is diagonal. For every $b \in E, T_{\lambda}(b)$ maps $\mathscr{M}_{l}$ into $\mathscr{M}_{l+1}$ if $l<k-1$ and it maps $\mathscr{M}_{k-1}$ into $\mathcal{M}_{0}$. Hence its matrix has non zero terms only on the first lower diagonal and in the $0, k-1$ entry.

Now write

$$
\mathscr{B}=\sum_{i=0}^{k-1} \sum_{j=0}^{k-1} S^{i} \mathscr{T}_{k} S^{* j} e_{i j}
$$

For $a \in A$ and $0 \leq l \leq k-1$, the $l, l$ entry of $\phi_{\infty}(a)$ is

$$
\begin{aligned}
\alpha^{l}(a) \delta_{l l}+\alpha^{l+k}(a) \delta_{l+k, l+k}+\cdots & =S^{l}\left(\alpha^{l}(a) \delta_{00}+\alpha^{k+l}(a) \delta_{k k}+\cdots\right) S^{* l} \\
& =S^{l} \phi_{\infty}^{(k)}\left(\alpha^{l}(a)\right) S^{* l} \in S^{l} \mathscr{T}_{k} S^{* l}
\end{aligned}
$$

For $b \in E$, the $l+1, l$ entry of $T_{\lambda}(b)$, for $l<k-1$, is

$$
\alpha^{l}\left(\lambda_{l+1} b\right) \delta_{l+1, l}+\alpha^{l+k}\left(\lambda_{l+1+k} b\right) \delta_{l+1+k, l+k}+\cdots
$$


Since $\lambda$ is of period $k$, this is equal to

$$
\begin{aligned}
& \alpha^{l}\left(\lambda_{l+1} b\right) \delta_{l+1, l}+\alpha^{k}\left(\alpha^{l}\left(\lambda_{l+1} b\right)\right) \delta_{l+1+k, l+k}+\cdots \\
& =S^{l+1}\left(\alpha^{l}\left(\lambda_{l+1} b\right) \delta_{00}+\alpha^{k+l}\left(\lambda_{l+1} b\right) \delta_{k, k}+\cdots\right) S^{* l} \\
& =S^{l+1} \phi_{\infty}^{(k)}\left(\alpha^{l}\left(\lambda_{l+1} b\right)\right) S^{* l} \in S^{l+1} \mathscr{T}_{k} S^{* l} .
\end{aligned}
$$

For the $0, k-1$ entry of $T_{\lambda}(b)$ we get

$$
\begin{aligned}
\left(\alpha^{k-1}\left(\lambda_{k} b\right) \delta_{k, 0}+\alpha^{2 k-1}\left(\lambda_{k} b\right)\right. & \left.\delta_{2 k, k}+\cdots\right) S^{*(k-1)} \\
& =T^{(k)}\left(\alpha^{k-1}\left(\lambda_{k} b\right)\right) S^{*(k-1)} \in \mathscr{T}_{k} S^{*(k-1)} .
\end{aligned}
$$

Now write $\mathscr{A}_{k}$ for the $\mathrm{C}^{*}$-algebra generated by all the periodic representations of period $k$. It follows from the above that $\mathscr{A}_{k} \subseteq \mathscr{B}$. We shall now show that, in fact, the two algebras are equal.

Fix $0 \leq j<k-1$. Setting $\lambda_{i}=1$ if $i \equiv j+1(\bmod k)$ and $\lambda_{i}=0$ otherwise, we find, using (1) and (2), that $S^{j+1} \phi_{\infty}^{(k)}\left(\alpha^{j}(b)\right) S^{* j} e_{j+1, j}=T_{\lambda}(b) \in \mathscr{A}_{k}$. Since this holds for all $b$ in $A$, we conclude that, for all $a \in A$ and every $0 \leq j<k-1$,

$$
S^{j+1} \phi_{\infty}^{(k)}(a) S^{* j} e_{j+1, j} \in \mathscr{A}_{k} .
$$

Similarly, by setting $\lambda_{i}=1$ if $i \equiv 0(\bmod k)$ and $\lambda_{i}=0$ otherwise, we find that

$$
T^{(k)}(E) S^{*(k-1)} e_{0, k-1} \subseteq \mathscr{A}_{k} .
$$

Thus, for every $b, a_{1}, \ldots a_{k-1}$ in $A$ we find that $T^{(k)}(b) S^{*(k-1)} e_{0, k-1}$, $S^{k-1} \phi_{\infty}^{(k)}\left(a_{k-1}\right) S^{*(k-2)} e_{k-1, k-2}, \ldots$ and $S \phi_{\infty}^{(k)}\left(a_{1}\right) e_{1,0}$ all lie in $\mathscr{A}_{k}$. Multiplying them, we see that $T^{(k)}(b) \phi_{\infty}^{(k)}\left(a_{k-1} \ldots a_{1}\right) e_{0,0}$ lie in $\mathscr{A}_{k}$.

Thus $T^{(k)}(E) e_{0,0} \subseteq \mathscr{A}_{k}$. Since $\left\{X: X e_{0,0} \in \mathscr{A}_{k}\right\}$ is a $\mathrm{C}^{*}$-algebra,

$$
\mathscr{T}_{k} e_{0,0} \subseteq \mathscr{A}_{k} .
$$

Setting, in (3), $a=1$ we find that $S^{j+1} S^{* j} e_{j+1, j}$ lies in $\mathscr{A}_{k}$ (for $0 \leq j<k-1$ ). It follows that

$$
S^{j+1} e_{j+1,0}=\left(S^{j+1} S^{* j} e_{j+1, j}\right)\left(S^{j} S^{*(j-1)} e_{j, j-1}\right) \ldots S e_{1,0} \in \mathscr{A}_{k} .
$$

Finally, for $0 \leq i, j \leq k-1$ and $X$ in $\mathscr{T}_{k}$,

$$
S^{j} X S^{* i} e_{i, j}=\left(S^{j} e_{j, 0}\right)\left(X e_{0,0}\right)\left(S^{i} e_{i, 0}\right)^{*} \in \mathscr{A}_{k} .
$$

Hence $\mathscr{B}=\mathscr{A}_{k}$. But $\mathscr{B}$ is clearly isomorphic to $M_{k}\left(\mathscr{T}_{k}\right)$ and the isomorphism is given by conjugating by the (isometric) matrix $\operatorname{diag}\left(1, S, S^{2}, \ldots, S^{k-1}\right)$. 
Suppose $n, m$ are two natural numbers with $n \mid m$ and let $\lambda$ be periodic of period $n$. Then $\lambda$ is periodic of period $m$ and this implies that $\mathscr{A}_{n} \subseteq \mathscr{A}_{m}$. Using the isomorphisms of Lemma 2.3 we get an injective *-homomorphism $\beta_{n, m}: M_{n}\left(\mathscr{T}_{n}\right) \rightarrow M_{m}\left(\mathscr{T}_{m}\right)$. In the next result we describe this map. In what follows we keep the notation set up in the proof of Lemma 2.3.

Lemma 2.4. Let $n, m$ be two natural numbers with $n \mid m$. Write $k=m / n$. Then the restriction of $\beta_{m, n}$ to $\mathscr{T}_{n} e_{0,0}$ is defined by

$$
\beta_{n, m}\left(\phi_{\infty}^{(n)}(a) e_{0,0}\right)=\sum_{j=0}^{k-1} \phi_{\infty}^{(m)}\left(\alpha^{j n}(a)\right) e_{j n, j n}
$$

for $a \in A$ and

$$
\beta_{n, m}\left(T^{(n)}(b) e_{0,0}\right)=\sum_{j=0}^{k-2} \phi_{\infty}^{(m)}\left(\alpha^{j n}(b)\right) e_{(j+1) n, j n}+T^{(m)}\left(\alpha^{(k-1) n}(b)\right) e_{0,(k-1) n}
$$

for $b \in E$.

Also, for $0 \leq i, j \leq n-1$ we have

$$
\beta_{n, m}\left(e_{i, j}\right)=\sum_{l=0}^{k-1} e_{i+l n, j+l n}
$$

Proof. In the following we shall write $\eta_{n}$ for the isomorphism $\eta_{n}: \mathscr{A}_{n} \rightarrow$ $M_{n}\left(\mathscr{T}_{n}\right)$ of Lemma 2.3. Fix $0 \leq j<n-1$ and let $\lambda_{p}$ be 1 if $p \equiv j+1(\bmod n)$ and be 0 otherwise. Then (by equations (1) and (2)) the matricial form of $T_{\lambda}(1)$ as an element of $\mathscr{A}_{n}$ is $S^{j+1} S^{* j} e_{j+1, j}$. Thus $\eta_{n}\left(T_{\lambda}(1)\right)=e_{j+1, j}$. Using (1) and (2) again, this time with $m$ instead of $n$, we find that the matricial form of $T_{\lambda}(1)$ as an element of $\mathscr{A}_{m}$ is $\sum_{l=0}^{k-1} S^{j+n l+1} S^{*(j+n l)} e_{j+n l+1, j+n l}$ and, thus, $\eta_{m}\left(T_{\lambda}(1)\right)=\sum e_{j+n l+1, j+n l}$. Equation (6) for $i=j+1$ follows. Since $\beta_{n, m}$ is a $*$-homomorphism, (6) follows for all $i, j$.

Now let $\lambda$ be defined by $\lambda_{p}=1$ if $p$ is a multiple of $n$ and is equal to 0 otherwise. Then, for $b \in E$, the matricial form of $T_{\lambda}\left(\alpha^{1-n}(b)\right)$ as an element of $\mathscr{A}_{n}$ is $T^{(n)}(b) S^{*(n-1)} e_{0, n-1}$ (hence $\left.\eta_{n}\left(T_{\lambda}\left(\alpha^{1-n}(b)\right)\right)=T^{(n)}(b) e_{0, n-1}\right)$ and its matricial form as an element of $\mathscr{A}_{m}$ is $T^{(m)}\left(\alpha^{(k-1) n}(b)\right) S^{*(m-1)} e_{0, m-1}+$ $\sum_{l=1}^{k-1} S^{l n} \phi_{\infty}^{(m)}\left(\alpha^{l-1}(b)\right) S^{*(l n-1)} e_{l n, l n-1}$ (and therefore $\eta_{m}\left(T_{\lambda}\left(\alpha^{1-n}(b)\right)\right)=$ $\left.T^{(m)}\left(\alpha^{(k-1) n}(b)\right) e_{0, m-1}+\sum_{l=1}^{k-1} \phi_{\infty}^{(m)}\left(\alpha^{l-1}(b)\right) e_{l n, l n-1}\right)$. It follows that

$$
\beta_{n, m}\left(T^{(n)}(b) e_{0, n-1}\right)=T^{(m)}\left(\alpha^{(k-1) n}(b)\right) e_{0, m-1}+\sum_{l=1}^{k-1} \phi_{\infty}^{(m)}\left(\alpha^{l-1}(b)\right) e_{l n, l n-1} .
$$


Since

$$
T^{(n)}(b) e_{0,0}=\left(T^{(n)}(b) e_{0, n-1}\right) \cdot e_{n-1, n-2} \ldots e_{1,0}
$$

and,

$$
\phi_{\infty}^{(n)}(a) e_{0,0}=\left(T^{(n)}(1) e_{0,0}\right)^{*} T^{(n)}(a) e_{0,0}, \quad a \in A,
$$

a straightforward computation, using the fact that $\beta_{n, m}$ is a *-homomorphism, completes the proof.

COROLlaRY 2.5. For $n \mid m$ the map $\beta_{n, m}$, described above, maps $\mathscr{T}_{n} e_{i, j}$ into $\sum_{l=0}^{k-1} \mathscr{T}_{m} e_{i+\ln , j+\ln }$ where $k=m / n$.

In fact, define the map $\theta_{n, m}: \mathscr{T}_{n} \rightarrow M_{k}\left(\mathscr{T}_{m}\right)$ by

$$
\theta_{n, m}\left(\phi_{\infty}^{(n)}(a)\right)=\sum_{j=0}^{k-1} \phi_{\infty}^{(m)}\left(\alpha^{j n}(a)\right) e_{j, j}
$$

for $a \in A$ and

$$
\theta_{n, m}\left(T^{(n)}(b)\right)=T^{(m)}\left(\alpha^{(k-1) n}(b)\right) e_{0, k-1}+\sum_{j=0}^{k-2} \phi_{\infty}^{(m)}\left(\alpha^{j}(b)\right) e_{j+1, j}
$$

for $b \in E$.

The matricial form of the elements of $\mathscr{A}_{m}$ was with respect to the decomposition

$$
\mathscr{F}=\mathcal{M}_{0} \oplus \cdots \oplus \mathcal{M}_{m-1}
$$

where $\mathscr{M}_{l}=E^{l} \otimes \mathscr{F}_{m}$. As a $C^{*}$-module over $A$ each $\mathscr{M}_{l}$ is isomorphic to $\mathscr{F}_{m}$. Thus any permutation of these spaces defines a unitary operator on $\mathscr{F}$. Let $U$ be the operator arising from the permutation $(0,1, \ldots, m-1) \mapsto$ $(0, n, \ldots,(k-1) n, 1,2, \ldots, m-n-1, m-1)$. Then we have the following.

COROllary 2.6. With the notation above,

$$
\beta_{n, m}=U^{*}\left(I_{k} \otimes \theta_{n, m}\right) U
$$

The following lemma follows immediately from the definition of the maps $\beta_{n, m}$ (or by a straightforward tedious computation using Lemma 2.4).

LeMмa 2.7. For positive integers $n, k, l$ we have

$$
\beta_{n k, n k l} \circ \beta_{n, n k}=\beta_{n, n k l} .
$$

It is known that the Toeplitz algebra $\mathscr{T}_{n}$ is an extension of the crossed product algebra of $A$ by $\alpha^{n}, A \times_{\alpha^{n}}$ Z. The Toeplitz algebra contains the algebra 
$K\left(\mathscr{F}_{n}\right)$ of all compact operators on the $\mathrm{C}^{*}$-module $\mathscr{F}_{n}$ (where "compact" is in the sense of $\mathrm{C}^{*}$-modules theory) which is isomorphic to $A \otimes K$ (where $K$ denotes the algebra of compact operators on a separable Hilbert space). The quotient space $\mathscr{T}_{n} / K\left(\mathscr{F}_{n}\right)$ is then isomorphic to the crossed product. One can also check (see e.g. [6]) that the ideal $K\left(\mathscr{F}_{n}\right)$ is generated by the set $\phi_{\infty}^{(n)}(A) P_{0}$ where $P_{0}$ is the projection of $\mathscr{F}_{n}$ onto the first summand of $\mathscr{F}_{n}$ (i.e. $A$ ).

We now compute, for $a, b, c$ in $A$ and $k \geq 1$,

$$
\begin{aligned}
\left(\phi_{\infty}\left(a c^{*}\right)-T(\alpha(a)) T(\alpha(c))^{*}\right) \delta_{k} b & =\delta_{k} \alpha^{k}\left(a c^{*}\right) b-T(\alpha(a)) \delta_{k-1} \alpha^{k}\left(c^{*}\right) b \\
& =\delta_{k} \alpha^{k}\left(a c^{*}\right) b-\delta_{k} \alpha^{k}\left(a c^{*}\right) b=0
\end{aligned}
$$

and, for $k=0$,

$$
\left(\phi_{\infty}\left(a c^{*}\right)-T(\alpha(a)) T(\alpha(c))^{*}\right) \delta_{0} b=\delta_{0} a c^{*} b .
$$

Hence,

$$
\phi_{\infty}\left(a c^{*}\right)-T(\alpha(a)) T(\alpha(c))^{*}=\phi_{\infty}\left(a c^{*}\right) P_{0} .
$$

Lemma 2.8. For every positive integers $n, m$ with $m / n=k \in \mathrm{Z}$ the map $\beta_{n, m}$ induces a map, denoted $\gamma_{n, m}$, from $M_{n}\left(A \times_{\alpha^{n}} Z\right)$ into $M_{m}\left(A \times_{\alpha^{m}} \mathrm{Z}\right)$.

PROOF. Using Corollary 2.6 it suffices to show that a similar statement holds for the map $\theta_{n, m}$. For this, we have to show that $\theta_{n, m}$ maps $K\left(\mathscr{F}_{n}\right)$ into $M_{k}\left(K\left(\mathscr{F}_{m}\right)\right)$. We compute, for $a, c \in A$,

$$
\begin{aligned}
\theta_{n, m}( & \left.\phi_{\infty}^{(n)}\left(a c^{*}\right)-T^{(n)}\left(\alpha^{n}(a)\right) T^{(n)}\left(\alpha^{n}(c)\right)^{*}\right) \\
= & \sum_{j=0}^{k-1} \phi_{\infty}^{(m)}\left(\alpha^{j n}\left(a c^{*}\right)\right) e_{j, j}-T^{(m)}\left(\alpha^{(k-1) n}\left(\alpha^{n}(a)\right)\right) T^{(m)}\left(\alpha^{(k-1) n}\left(\alpha^{n}(c)\right)\right)^{*} e_{0,0} \\
& \quad-\sum_{j=0}^{k-2} \phi_{\infty}^{(m)}\left(\alpha^{j n}\left(\alpha^{n}\left(a c^{*}\right)\right)\right) e_{j+1, j+1} \\
= & \left(\phi_{\infty}^{(m)}\left(a c^{*}\right)-T^{(m)}\left(\alpha^{m}(a)\right) T^{(m)}\left(\alpha^{m}(c)\right)^{*}\right) e_{0,0} .
\end{aligned}
$$

From the discussion preceeding the lemma it now follows that $\theta_{n, m}$ maps the set $\phi_{\infty}^{(n)}(A) P_{0}$ into $M_{k}\left(K\left(\mathscr{F}_{m}\right)\right)$. Since this set generates the ideal $K\left(\mathscr{F}_{n}\right)$, the claim follows.

Let $\gamma_{m, n}$ be the map defined above and let $q_{n}$ and $q_{m}$ be the quotient maps on $M_{n}\left(\mathscr{T}_{n}\right)$ and $M_{m}\left(\mathscr{T}_{m}\right)$ respectively (induced by $q$ ). Recall that $q\left(\phi_{\infty}^{(n)}(a)\right)=a$ for $a \in A$ and $q\left(T^{(n)}(1)\right)=u_{n}^{*}$ (where $u_{n}$ is the unitary that, together with $A$, 
generates $A \times \alpha_{\alpha^{n}} \mathrm{Z}$ and satisfies $\left.u_{n} a u_{n}^{*}=\alpha^{n}(a), a \in A\right)$. Then it follows from the definition of $\beta_{n, m}$ that $\gamma_{n, m}$ is defined by

$$
\gamma_{n, m}\left(a e_{0,0}\right)=\sum_{l=0}^{k-1} \alpha^{l n}(a) e_{l n, l n}
$$

for $a \in A$,

$$
\gamma_{n, m}\left(u_{n}\right)=u_{m} e_{(k-1) n, 0}+\sum_{l=0}^{k-2} e_{l n,(l+1) n}
$$

and, for $0 \leq i, j \leq n-1$,

$$
\gamma_{n, m}\left(e_{i, j}\right)=\sum_{l=0}^{k-1} e_{i+\ln , j+\ln } .
$$

We now write $\mathscr{B}(n)$ (or $\mathscr{B}_{\alpha}(n)$ if we want to emphasize the dependence on $\alpha$ ) for the algebra $M_{n}\left(A \times_{\alpha^{n}} \mathrm{Z}\right)$.

DEFINITION 2.9. Suppose that $\left\{n_{k}\right\}$ is an increasing sequence of positive integers such that $n_{k}$ divides $n_{k+1}$ for $k \geq 1$. The Bunce-Deddens algebra of $\alpha$ is the direct limit

$$
\mathscr{B}_{\alpha}\left(\left\{n_{k}\right\}\right)=\lim _{\rightarrow}\left(\mathscr{B}\left(n_{k}\right), \gamma_{n_{k}, n_{k+1}}\right)
$$

Recall that an element $\sum_{k=-\infty}^{\infty} a_{k} u_{n}^{k}$ in the crossed product is 0 if and only if $a_{k}=0$ for every $k$. It follows from this and the definition of $\gamma_{n, m}$ that this map is injective. We can, thus, write the limit algebra $\mathscr{B}_{\alpha}\left(\left\{n_{k}\right\}\right)$ as the closure of the increasing union $\cup \mathscr{B}\left(n_{k}\right)$.

Note that the analysis above shows that, for every $k \geq 1$, we have

$$
\mathscr{B}\left(n_{k}\right)=M_{n_{k}}\left(A \times_{\alpha^{n_{k}}} \mathrm{Z}\right) \cong M_{n_{k}}\left(\mathscr{T}_{n_{k}}\right) / M_{n_{k}}\left(K\left(\mathscr{F}_{n_{k}}\right)\right) \cong \mathscr{A}_{n_{k}} / K(\mathscr{F}) .
$$

It follows that $\mathscr{B}_{\alpha}\left(\left\{n_{k}\right\}\right)$ is isomorphic to the quotient of $\overline{\cup \mathscr{A}_{n_{k}}}$ by $K(\mathscr{F})$. Recall that $\overline{\cup \mathscr{A}_{n_{k}}}$ is the $\mathrm{C}^{*}$-algebras generated by all periodic representations of perion $n_{k}$ for all $k \geq 1$.

Proposition 2.10. Let $\left\{n_{k}\right\}$ and $\left\{m_{j}\right\}$ be increasing sequences of positive integers for which $n_{k} \mid n_{k+1}$ and $m_{j} \mid m_{j+1}$ for all $j, k \geq 1$. Write $\delta\left(n_{k}\right)$ and $\delta\left(m_{j}\right)$ for the corresponding supernatural numbers. If $\delta\left(n_{k}\right)=\delta\left(m_{j}\right)$ then $\mathscr{B}_{\alpha}\left(\left\{n_{k}\right\}\right)$ and $\mathscr{B}_{\alpha}\left(\left\{m_{j}\right\}\right)$ are isomorphic.

Proof. The proof is standard. For each $k, n_{k}$ divides some $m_{r}$ and the map $\gamma_{n_{k}, m_{r}}$ is the inclusion of $\mathscr{B}\left(n_{k}\right)$ into $\mathscr{B}\left(m_{r}\right)$. Since the latter algebra is 
contained in $\mathscr{B}\left(\left\{m_{j}\right\}\right)$, we find that every $\mathscr{B}\left(n_{k}\right)$ is contained there. Since this holds for all $k, \mathscr{B}\left(\left\{n_{k}\right\}\right) \subseteq \mathscr{B}\left(\left\{m_{j}\right\}\right)$. Equality follows from symmetry.

The converse of the proposition above holds in some cases (see Corollary 4.2) but not in general, as we see in the following lemma.

Lemma 2.11. Suppose A is a unital $C^{*}$-algebra such that $A$ is isomorphic to $M_{p}(A)$ for some positive integer $p$. Let $\left\{n_{k}\right\}$ be an increasing sequence as above and let $\alpha=\mathrm{id}$ (the identity automorphism). Then

$$
\mathscr{B}_{\mathrm{id}}\left(\left\{n_{k}\right\}\right) \cong \mathscr{B}_{\mathrm{id}}\left(\left\{p n_{k}\right\}\right) .
$$

Proof. Since $\alpha=\mathrm{id}, A \times_{\alpha^{n}} \mathrm{Z} \cong A \otimes C(\mathrm{~T})$ for all $n$. Making these identifications, the maps $\gamma_{n_{k}, n_{k+1}}$ (for the algebra $\mathscr{B}_{\text {id }}\left(\left\{n_{k}\right\}\right)$ ) now satisfy (when we write $m_{k}$ for $n_{k+1} / n_{k}$ )

$$
\gamma_{n_{k}, n_{k+1}}\left((a \otimes 1) e_{i, j}\right)=\sum_{l=0}^{m_{k}-1}(a \otimes 1) e_{i+l n_{k}, j+l n_{k}}
$$

for $a \in A$, and

$$
\gamma_{n_{k}, n_{k+1}}\left((a \otimes z) e_{i, j}\right)=(a \otimes z) e_{i+\left(m_{k}-1\right) n_{k}, j}+\sum_{l=0}^{m_{k}-2}(a \otimes 1) e_{i+n_{k} l, j+n_{k}(l+1)}
$$

where $z$ is the identity function on T. The maps $\gamma_{p n_{k}, p n_{k+1}}$ (for the other algebra) can be written similarly. Write $\psi$ for the isomorphism $\psi: A \rightarrow M_{p}(A)$. It induces an isomorphism from $A \otimes C(\mathrm{~T})$ onto $M_{p}(A \otimes C(\mathrm{~T}))$ defined by

$$
t(a \otimes f)=\sum_{0 \leq i, j \leq p-1}\left(\psi(a)_{i, j} \otimes f\right) e_{i, j} .
$$

For every positive integer $k$ we write $t_{k}$ for the isomorphism from $M_{k}(A \otimes C(\mathrm{~T}))$ onto $M_{k p}(A \otimes C(\mathrm{~T}))$ defined by applying $t$ to each entry. Hence, for $0 \leq r, q \leq$ $k-1$,

$$
t_{k}\left((a \otimes f) e_{r, q}\right)=\sum_{0 \leq i, j \leq p-1}\left(\psi(a)_{i, j} \otimes f\right) e_{i+r p, j+q p} .
$$

To prove the claimed isomorphism it suffices to show that, for every $k \geq 1$,

$$
\gamma_{p n_{k}, p n_{k+1}} \circ t_{n_{k}}=t_{n_{k+1}} \circ \gamma_{n_{k}, n_{k+1}} .
$$

The required isomorphism in this case is $\lim _{\rightarrow} t_{n_{k}}$. To prove (8) it is enough to apply both sides to elements of the form $(a \otimes z) e_{0,0}$ and $(a \otimes 1) e_{r, q}$ for $a \in A$. The computation is straightforward and is omitted. 


\section{The structure of $\mathscr{B}_{\alpha}\left(\left\{n_{k}\right\}\right)$}

Proposition 3.1. $\mathscr{B}_{\alpha}\left(\left\{n_{k}\right\}\right)$ has a unique tracial state $\tau$ if and only if $A$ has an $\alpha$-invariant tracial state $\tau_{0}$ and for every $k \geq 1$ this is the unique $\alpha^{n_{k}}$-invariant tracial state on A.

Moreover, in this case, $\tau$ is faithful if and only if $\tau_{0}$ is.

Proof. Let $\tau_{0}$ be an $\alpha^{n_{l}}$-invariant tracial state on $A$. For every $k \geq 1$, let $\mathscr{E}_{k}$ be the canonical conditional expectation of the crossed product $A \times{ }_{\alpha^{n_{k}}} \mathrm{Z}$ onto $A$ and write $\tau_{k}=\tau_{0} \circ \mathscr{E}_{k}$. If $k \geq l$ then $\tau_{k}$ is a tracial state on the crossed product (faithful if $\tau_{0}$ is). For such $k$ and an element $X=\left(x_{i j}\right)$ of the $n_{k} \times n_{k}$ matrices over the crossed product $A \times_{\alpha}^{n_{k}} Z$, we set $\tilde{\tau}_{k}(X)=1 / n_{k}\left(\sum \tau_{k}\left(x_{i i}\right)\right)$ and this defines a tracial state on $\mathscr{B}\left(n_{k}\right)$ (that is faithful if $\tau_{0}$ is). To show that these traces define a tracial state on the direct limit it is left to show that for every $k \geq l$,

$$
\tilde{\tau}_{k+1} \circ \beta_{n_{k}, n_{k+1}}=\tilde{\tau}_{k} .
$$

For $X=a e_{0,0}$ we have (set $m=n_{k+1} / n_{k}$ ),

$$
\begin{aligned}
\tilde{\tau}_{k+1} \circ \gamma_{n_{k}, n_{k+1}}\left(a e_{0,0}\right) & =\tilde{\tau}_{k+1}\left(\sum_{j=0}^{m-1} \alpha^{j n_{k}}(a) e_{j n_{k}, j n_{k}}\right)=\sum 1 / n_{k+1} \tau_{k}\left(\alpha^{j n_{k}}(a)\right) \\
& =1 / n_{k} \tau_{0}(a)=\tilde{\tau}_{k}\left(a e_{0,0}\right) .
\end{aligned}
$$

For $X=u_{n_{k}} e_{0,0}$ we have,

$$
\begin{aligned}
\tilde{\tau}_{k+1} \circ \gamma_{n_{k}, n_{k+1}}\left(u_{n_{k}} e_{0,0}\right) & =\tilde{\tau}_{k+1}\left(u_{n_{k+1}} e_{0,(k-1) n_{k}}+\sum \tilde{\tau}_{k+1}\left(e_{(j+1) n_{k}, j n_{k}}\right)\right. \\
& =0=\tau_{k}\left(u_{n_{k}}\right)=\tilde{\tau}_{k}\left(u_{n_{k}} e_{0,0}\right) .
\end{aligned}
$$

Since every tracial state on $M_{n_{k}}\left(A \times_{\alpha^{n_{k}}} Z\right)$ is determined by its values on elements of the form $X e_{0,0}\left(X \in A \times_{\alpha^{n_{k}}} Z\right.$ Z), this proves (9). Thus, we constructed a tracial state $\tau$ on $\mathscr{B}_{\alpha}\left(\left\{n_{k}\right\}\right)$ that is faithful if $\tau_{0}$ is. Write $\tau=\Phi_{l}\left(\tau_{0}\right)$. So $\Phi_{l}$ maps $\alpha^{n_{l}}$-invariant traces of $A$ to traces of $\mathscr{B}_{\alpha}\left(\left\{n_{k}\right\}\right)$. It follows from the definition of $\Phi_{l}$ that it is one-to-one.

To go in the other direction, fix a tracial state $\tau$ on $\mathscr{B}_{\alpha}\left(\left\{n_{k}\right\}\right)$ and define, for $y$ in $A \times_{\alpha^{n_{k}}} \mathrm{Z}, \tau_{k}(y)=1 / n_{k} \tau\left(y e_{0,0}\right)$ (where $e_{0,0}$ is an element of $\mathscr{B}\left(n_{k}\right)$ ). Note that we have the following.

$$
\begin{aligned}
\tau_{k}\left(u_{n_{k}}\right) & =1 / n_{k} \tau\left(u_{n_{k}} e_{0,0}\right)=1 / n_{k} \tau\left(\gamma_{n_{k}, n_{k+1}}\left(u_{n_{k}} e_{0,0}\right)\right) \\
& =1 / n_{k}\left(\tau\left(u_{n_{k+1}} e_{n_{k+1}-n_{k}, 0}\right)-\sum_{l=0}^{m-2} \tau\left(e_{l n_{k},(l+1) n_{k}}\right)\right)=0
\end{aligned}
$$


where $m=n_{k+1} / n_{k}$. The last term is equal to 0 because we evaluate $\tau$ there on matrices whose diagonal entries are all zeros. It follows that $\tau_{k}=\left(\tau_{k} \mid A\right) \circ \mathscr{E}_{k}$ ; i.e. $\tau_{k}$ is determined by its values on $A$. Now set $\Psi_{k}(\tau)=\tau_{k} \mid A$ for $a \in A$. Since $\tau_{k}$ is a tracial state on $A \times_{\alpha^{n_{k}}} Z$, it follows that $\Psi_{k}(\tau)$ is $\alpha^{n_{k}}$-invariant.

Now suppose that $\mathscr{B}_{\alpha}\left(\left\{n_{k}\right\}\right)$ has a unique tracial state $\tau$. Let $\tau_{0}$ be $\Psi_{1}(\tau)$. Then it is an $\alpha$-invariant tracial state on $A$. Fix $k \geq 1$. Suppose $\tau_{0}^{\prime}$ is another (different) $\alpha^{n_{k}}$-invariant trace on $A$. Then $\Phi_{k}\left(\tau_{0}^{\prime}\right) \neq \Phi_{k}\left(\tau_{0}\right)$ (since $\Phi_{k}$ is injective). But this contradicts the assumed uniqueness of traces on $\mathscr{B}_{\alpha}\left(\left\{n_{k}\right\}\right)$. This proves one direction. Note also that if $\tau$ is faithful then so is $\tau_{0}$ (which is, roughly speaking, a restriction of $\tau$ to a copy of $A$ ).

For the other direction, let $\tau_{0}$ be an $\alpha$-invariant trace with the uniquness property stated in the proposition. Write $\tau=\Phi_{1}\left(\tau_{0}\right)$. Then $\tau$ is a tracial state on $\mathscr{B}_{\alpha}\left(\left\{n_{k}\right\}\right)$. Suppose $\tau^{\prime}$ ia also a tracial state on $\mathscr{B}_{\alpha}\left(\left\{n_{k}\right\}\right)$ that is different from $\tau$. But then, for some $k \geq 1$, the restrictions of $\tau$ and $\tau^{\prime}$ to $\mathscr{B}\left(n_{k}\right)$ are different. Since a trace on $\mathscr{B}\left(n_{k}\right)=M_{n_{k}}\left(A \times_{\alpha^{n_{k}}} Z\right)$ is determined by its values on matrices of the form $y e_{0,0}$ for $y \in A \times_{\alpha^{n_{k}}} Z$, and since, as we saw above, these values are determined by the restriction to elements of the form $a e_{0,0}$ for $a \in A$, we find that $\Psi_{k}(\tau) \neq \Psi_{k}\left(\tau^{\prime}\right)$. But this contradicts the uniqueness property of $\tau_{0}$. In the construction of $\Phi_{1}\left(\tau_{0}\right)$ above it was noted that if $\tau_{0}$ is faithful so is $\Phi_{1}\left(\tau_{0}\right)$.

We shall now proceed to show that the algebra $\mathscr{B}_{\alpha}\left(\left\{n_{k}\right\}\right)$ is isomorphic to a crossed product algebra of the form $C(X, A) \times_{\sigma} \mathrm{Z}$.

In order to define the space $X$, fix a sequence $\left\{n_{k}\right\}$ of positive integers with $n_{k} \mid n_{k+1}$ for all $k \geq 1$ and with $n_{1}=1$. Set $m_{k}=n_{k+1} / n_{k}$, let $X_{i}=$ $\left\{0,1, \ldots, m_{i}-1\right\}$ and let $X$ be the Cantor set $X=\prod_{i=1}^{\infty} X_{i}$. We can think of each element $x$ of $X$ either as a sequence $\left\{x_{i}\right\}$ or as a formal sum $\sum_{i} x_{i} n_{i}$. We write $\sigma_{0}$ for the odometer action on $X$. For $x \in X$ let $i(x)$ be the smallest $i$ for which $x_{i}<m_{i}-1$ (and $i(x)=\infty$ if $x_{i}=m_{i}-1$ for all $i$ ). Then, if $i(x)<\infty, \sigma_{0}(x)_{i}=0$ for all $i<i(x), \sigma_{0}(x)_{i(x)}=x_{i(x)}+1$ and $\sigma_{0}(x)_{i}=x_{i}$ for $i>i(x)$. If $i(x)=\infty, \sigma_{0}(x)=\{0,0, \ldots\}$. It is known that this map is a homoemorphism on $X$.

We consider the cylinder sets

$$
J\left(x_{1}, x_{2}, \ldots x_{k}\right)=\left\{y \in X: y_{i}=x_{i} 1 \leq i \leq k\right\} .
$$

Then $\sigma_{0}\left(J\left(x_{1}, \ldots, x_{k}\right)\right)=J\left(y_{1}, \ldots, y_{k}\right)$ where

$$
1+\sum_{i=1}^{k} x_{i} n_{i} \equiv \sum_{i=1}^{k} y_{i} n_{i} \quad\left(\bmod n_{k+1}\right) .
$$

Given $\left(y_{1}, \ldots, y_{k}\right)$ with $0 \leq y_{i} \leq m_{i}-1$, we write $\delta_{\left(y_{1}, \ldots, y_{k}\right)}$ for the function 
in $C(X, A)$ which is 1 (the unit of $A)$ on $J\left(y_{1}, \ldots, y_{k}\right)$ and 0 otherwise. For an element $a \in A$ we shall write (by a slight abuse of notation) also $a$ for the function in $C(X, A)$ that is constantly equal to $a$. For $k \geq 2$ write

$$
\begin{aligned}
\mathscr{C}_{k}=\{f \in C(X, A): f(x) & =f(y) \\
& \text { whenever } \left.\left(x_{1}, \ldots, x_{k-1}\right)=\left(y_{1}, \ldots, y_{k-1}\right)\right\}
\end{aligned}
$$

and, for $k=1, \mathscr{C}_{1}=A$ (where $A$ is viewed as the algebra of all constant functions in $C(X, A)$ ). Then $\mathscr{C}_{k} \subseteq \mathscr{C}_{k+1}$ and

$$
C(X, A)=\overline{\cup_{k=1}^{\infty} \mathscr{C}_{k}}
$$

Now define the automorphism $\sigma$ on $C(X, A)$ by

$$
\sigma(f)(x)=\alpha\left(f\left(\sigma_{0}^{-1}(x)\right)\right) .
$$

We shall show that $\mathscr{B}_{\alpha}\left(\left\{n_{k}\right\}\right)$ is isomorphic to $C(X, A) \times_{\sigma}$ Z. To do this, we write $U$ for the unitary that, together with $C(X, A)$, generates the crossed product and let, for every $k \geq 1, \mathscr{G}_{k}$ be the $\mathrm{C}^{*}$-algebra generated by $\mathscr{C}_{k}$ and $U$. For a given $k$ and $0 \leq j \leq n_{k}-1$, write $\mathbf{j}$ for $\left(j_{1}, \ldots, j_{k-1}\right)$ satisfying $\sum_{l} j_{l} n_{l}=j$. Note that we have

$$
U^{j} \delta_{\mathbf{0}} U^{* j}=\sigma^{j}\left(\delta_{\mathbf{0}}\right)=\delta_{\mathbf{0}} \circ \sigma_{0}^{-j}=\delta_{\mathbf{j}}
$$

for $0 \leq j \leq n_{k}-1$. For $j=n_{k}$ a similar argument shows that

$$
U^{n_{k}} \delta_{\mathbf{0}} U^{* n_{k}}=\delta_{\mathbf{0}} .
$$

Lemma 3.2. The algebra $\mathscr{G}_{k}$ is the $C^{*}$-algebra generated by the algebra $A$ (as a subalgebra of $\mathscr{C}_{k}$ ), the operator $\delta_{0} U^{n_{k}} \delta_{0}$ and the operators of the form $U^{i} \delta_{\mathbf{0}} U^{* j}$ for $0 \leq i, j \leq n_{k}-1$. (For $k=1$ we let $\delta_{\mathbf{0}}$ be 1$)$.

Proof. Write $\mathscr{D}_{k}$ for the algebra defined in the statement of the lemma. Since the function $\delta_{\mathbf{0}}$ lies in $\mathscr{C}_{k}$, it follows that $\mathscr{D}_{k} \subseteq \mathscr{G}_{k}$. Given $f \in \mathscr{C}_{k}$ and $0 \leq j \leq n_{k}-1$ we write $a_{j}=f\left(j_{1}, j_{2}, \ldots\right)$ where $j=\sum_{l=1}^{k-1} j_{l} n_{l}$. Then

$$
f=\sum_{j=0}^{n_{k}-1} a_{j} \delta_{\mathbf{j}}=\sum_{j} a_{j} U^{j} \delta_{\mathbf{0}} U^{* j} .
$$


Thus $\mathscr{C}_{k} \subseteq \mathscr{D}_{k}$. Also

$$
\begin{aligned}
U=\sum_{j=0}^{n_{k}-1} U \delta_{\mathbf{j}} & =\sum_{j=0}^{n_{k}-2} U\left(U^{j} \delta_{\mathbf{0}} U^{j *}\right)+U\left(U^{n_{k}-1} \delta_{\mathbf{0}} U^{\left(n_{k}-1\right) *}\right) \\
& =\sum_{j=0}^{n_{k}-2} U^{j+1} \delta_{\mathbf{0}} U^{j *}+\left(\delta_{\mathbf{0}} U^{n_{k}} \delta_{\mathbf{0}}\right)\left(\delta_{\mathbf{0}} U^{\left(n_{k}-1\right) *}\right) .
\end{aligned}
$$

Thus $U \in \mathscr{D}_{k}$, completing the proof.

Lemma 3.3. Fix $k \geq 1$ and define the map

$$
\rho_{k}: M_{n_{k}}\left(A \times_{\alpha^{n_{k}}} \mathbf{Z}\right) \rightarrow \mathscr{G}_{k}
$$

by

$$
\rho_{k}\left(a u_{n_{k}}^{l} e_{i, j}\right)=U^{* i} a \delta_{\mathbf{0}} U^{j+n_{k} l} .
$$

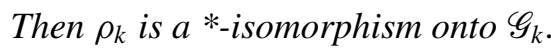

Proof. Note first that, since $a \delta_{\mathbf{0}}$ belongs to $\mathscr{C}_{k}$, the image of $\rho_{k}$ lies in $\mathscr{G}_{k}$. Write $x=a u_{n_{k}}^{l} e_{i, j}$ and $y=b u_{n_{k}}^{r} e_{p, q}$ for $a, b \in A, r, l \in \mathbf{Z}$ and $0 \leq$ $i, j, q, p \leq n_{k}-1$. We compute (assuming for simplicity that $j \geq p$ )

$$
\begin{aligned}
\rho_{k}(x) \rho_{k}(y) & =U^{* i} a \delta_{\mathbf{0}} U^{n_{k} l+j} U^{* p} b \delta_{\mathbf{0}} U^{n_{k} r+q} \\
& =U^{* i} a \delta_{\mathbf{0}} \alpha^{j-p+n_{k} l}(b) \delta_{\mathbf{j}-\mathbf{p}} U^{n_{k}(l+r)-p+j+q} .
\end{aligned}
$$

If $j \neq p, x y=0$ and the computation above shows that also $\rho_{k}(x) \rho_{k}(y)=0$ because $\delta_{\mathbf{0}} \delta_{\mathbf{j}-\mathbf{p}}=0$ in this case. Suppose $p=j$. Then the computation above shows that

$$
\begin{aligned}
\rho_{k}(x) \rho_{k}(y) & =U^{* i} a \alpha^{n_{k} l}(b) \delta_{\mathbf{0}} U^{n_{k}(l+r)} U^{q}=\rho_{k}\left(a \alpha^{n_{k} l}(b) u_{n_{k}}^{l+r} e_{i, q}\right) \\
& =\rho_{k}\left(a u_{n_{k}}^{l} b u_{n_{k}}^{r} e_{i, q}\right)=\rho_{k}(x y) .
\end{aligned}
$$

Thus $\rho_{k}$ is multiplicative. To show that $\rho_{k}$ is a $*$-map we compute

$$
\begin{aligned}
\left(\rho_{k}\left(a u_{n_{k}}^{l} e_{i, j}\right)\right)^{*} & =U^{-j-n_{k} l} \delta_{\mathbf{0}} a^{*} U^{i}=U^{* j} \alpha^{-n_{k} l}\left(a^{*}\right) \delta_{\mathbf{0}} U^{i-n_{k} l} \\
& =\rho_{k}\left(\alpha^{n_{k} l}\left(a^{*}\right) u_{n_{k}}^{* l} e_{j, i}\right)=\rho_{k}\left(\left(a u_{n_{k}}^{l} e_{i, j}\right)^{*}\right) .
\end{aligned}
$$

Note that

$$
\delta_{\mathbf{n}_{\mathbf{k}}-\mathbf{p}} U^{* i}\left(a \delta_{\mathbf{0}} U^{n_{k} l}\right) U^{j} \delta_{\mathbf{n}_{\mathbf{k}}-\mathbf{q}}=U^{* i}\left(a \delta_{\mathbf{0}} U^{n_{k} l}\right) U^{j}
$$

if $p=i$ and $q=j$ and it is equal 0 otherwise. Thus

$$
U^{p} \delta_{\mathbf{n}_{\mathbf{k}}-\mathbf{p}} \rho_{k}\left(\sum a_{i, j} u_{n_{k}}^{l_{i, j}} e_{i, j}\right) \delta_{\mathbf{n}_{\mathbf{k}}-\mathbf{q}} U^{* q}=a_{p, q} \delta_{\mathbf{0}} U^{n_{k} l_{p, q}} .
$$


Hence, if $\rho_{k}\left(\sum e_{i, j} u_{n_{k}}^{l_{i, j}} e_{i, j}\right)=0$, then $a_{i, j}=0$ for all $i, j$. Thus $\rho_{k}$ is injective. The fact that the map is onto follows from Lemma 3.2. (Note that $U^{i} \delta_{\mathbf{0}} U^{* j}=$ $\left.U^{*\left(n_{k}-i\right)} \delta_{\mathbf{0}} U^{n_{k}-j}=\rho_{k}\left(e_{n_{k}-i, n_{k}-j}\right)\right)$.

THEOREM 3.4. Let $\left\{n_{k}\right\}$ be an increasing sequence of positive integers with $n_{k} \mid n_{k+1}$ (and $n_{1}=1$ ). Then the algebra $\mathscr{B}_{\alpha}\left(\left\{n_{k}\right\}\right)$ is *-isomorphic to the algebra $C(X, A) \times_{\sigma} \mathrm{Z}$.

Proof. To construct the isomorphism we first show that, for every $k$,

$$
\rho_{k}=\rho_{k+1} \circ \gamma_{n_{k}, n_{k+1}} .
$$

Fix $k$ and write $m=n_{k} / n_{k+1}$. It will suffice to apply both maps to a generating set of $M_{n_{k}}\left(A \times_{\alpha^{n_{k}}} Z\right)$. In the following computations we shall write $\delta_{\mathbf{j}}^{(p)}$ instead of $\delta_{\mathbf{j}}$ as before, to indicate that $\mathbf{j}=\left(j_{1}, \ldots, j_{p-1}\right)$ is of length $p-1$ (and $\delta_{\mathbf{j}}^{(p)}$ is an element of $\mathscr{C}_{p}$ ). For $a \in A$ we have

$$
\begin{aligned}
& \rho_{k+1} \circ \gamma_{n_{k}, n_{k+1}}\left(a e_{0,0}\right)=\rho_{k+1}\left(\sum_{l=0}^{m-1} \alpha^{l n_{k}}(a) e_{l n_{k}, l n_{k}}\right) \\
& =\sum U^{* l n_{k}} \alpha^{n_{k} l}(a) \delta_{\mathbf{0}}^{(k+1)} U^{l n_{k}}=\sum a \delta_{\mathbf{n}_{\mathbf{k}+1}-\mathbf{n}_{\mathbf{k}}}^{(k+1)}=a \delta_{\mathbf{0}}^{(k)}=\rho_{k}\left(a e_{0,0}\right) .
\end{aligned}
$$

Also,

$$
\begin{gathered}
\rho_{k+1} \circ \gamma_{n_{k}, n_{k+1}}\left(u_{n_{k}} e_{0,0}\right)=\rho_{k+1}\left(u_{n_{k+1}} e_{(m-1) n_{k}, 0}\right)+\sum_{l=0}^{m-2} \rho_{k+1}\left(e_{l n_{k},(l+1) n_{k}}\right) \\
=U^{*(m-1) n_{k}} \delta_{\mathbf{0}}^{(k+1)} U^{n_{k+1}}+\sum_{l=0}^{m-2} U^{* l n_{k}} \delta_{\mathbf{0}}^{(k+1)} U^{(l+1) n_{k}} \\
=\delta_{\mathbf{n}_{\mathbf{k}}}^{(k+1)} U^{n_{k}}+\sum_{l=0}^{m-2} \delta_{\mathbf{n}_{\mathbf{k}+1}-\mathbf{n}_{\mathbf{k}}}^{(k+1)} U^{n_{k}}=\delta_{\mathbf{0}}^{(k)} U^{n_{k}}=\rho_{k}\left(u_{n_{k}} e_{0,0}\right) .
\end{gathered}
$$

For every $0 \leq i, j \leq n_{k}-1$,

$$
\begin{aligned}
\rho_{k} \circ \gamma_{n_{k}, n_{k+1}}\left(e_{i, j}\right)=\sum_{l=0}^{m-1} \rho_{k}\left(e_{i+l n_{k}, j+l n_{k}}\right)=\sum U^{*\left(i+l n_{k}\right)} \delta_{\mathbf{0}}^{(k+1)} U^{\left(j+l n_{k}\right)} \\
=\sum U^{* i} \delta_{\mathbf{n}_{\mathbf{k}+1}-\mathbf{l n}_{\mathbf{k}}}^{(k+1)} U^{j}=U^{* i} \delta_{\mathbf{0}}^{(k)} U^{j}=\rho_{k}\left(e_{i, j}\right) .
\end{aligned}
$$

This proves (10) and it follows that we have a *-homomorphism $\rho: \mathscr{B}_{\alpha}\left(\left\{n_{k}\right\}\right)$ $\rightarrow C(X, A) \times_{\sigma} \mathrm{Z}$ whose "restriction" to $\mathscr{B}\left(n_{k}\right)$ is $\rho_{k}$. Since each map $\rho_{k}$ is injective, so is $\rho$. It is left to show that $\rho$ is onto. Since the image of $\rho_{k}$ is $\mathscr{G}_{k}$, 
it amounts to showing that the (increasing) union of the algebras $\mathscr{G}_{k}$ is dense in the crossed product. But each of the algebras $\mathscr{G}_{k}$ contains $U$ and we know that $C(X, A)$ is the closure of the union of the algebras $\mathscr{C}_{k}$. Hence the density of $\cup \mathscr{G}_{k}$ follows.

THEOREM 3.5. Let $\left\{n_{k}\right\}$ be a sequence of positive integers with $n_{k} \mid n_{k+1}$ and $n_{1}=1$. Then the algebra $\mathscr{B}_{\alpha}\left(\left\{n_{k}\right\}\right)$ is simple if and only if, for every $k \geq 1, A$ is $\alpha^{n_{k}}$-simple (i.e. it has no proper closed two sided $\alpha^{n_{k}}$-invariant ideals).

Proof. Suppose that there is an $m \geq 1$ and an $\alpha^{n_{m}}$-invariant proper ideal $J \subseteq A$. Then $J$ is also $\alpha^{n_{k}}$-invariant for all $k \geq m$. For every such $k$, the closed ideal of $A \times_{\alpha^{n_{k}}} \mathrm{Z}$ generated by $J$ will be written $J_{k}$. Then $M_{n_{k}}\left(J_{k}\right)$ is an ideal (closed and proper) in $\mathscr{B}\left(n_{k}\right)$. It is easy to check (using the definition of $\gamma_{n_{k}, n_{k+1}}$ ) that

$$
\gamma_{n_{k}, n_{k+1}}\left(M_{n_{k}}\left(J_{k}\right)\right) \subseteq M_{n_{k+1}}\left(J_{k+1}\right)
$$

and, in fact,

$$
\gamma_{n_{k}, n_{k+1}}\left(M_{n_{k}}\left(J_{k}\right)\right)=M_{n_{k+1}}\left(J_{k+1}\right) \cap \gamma_{n_{k}, n_{k+1}}\left(M_{n_{k}}\left(A \times_{\alpha^{n_{k}}} \mathbf{Z}\right)\right) .
$$

Hence $\tilde{J}:=\lim _{\rightarrow} M_{n_{k}}\left(J_{k}\right)$ is a non zero ideal in $\mathscr{B}_{\alpha}\left(\left\{n_{k}\right\}\right)$. It also follows that, for $k \geq m, \tilde{J} \cap \mathscr{B}\left(n_{k}\right)=M_{n_{k}}\left(J_{k}\right)$. Thus $\tilde{J} \neq \mathscr{B}_{\alpha}\left(\left\{n_{k}\right\}\right)$.

We now turn to prove the other direction. We assume that $A$ is $\alpha^{n_{k}}$-simple for all $k \geq 1$. We start by showing that $C(X, A)$ is $\sigma$-simple. Let $I \subseteq C(X, A)$ be a $\sigma$-invariant ideal in $C(X, A)$. Since

$$
C(X, A)=\overline{\cup \mathscr{C}_{k}}
$$

where $\mathscr{C}_{k}$ (as defined above) form an increasing sequence of subalgebras, it follows that

$$
I=\overline{\cup\left(I \cap \mathscr{C}_{k}\right)} .
$$

Write $I_{k}=I \cap \mathscr{C}_{k}$. The algebra $\mathscr{C}_{k}$ can be identified with $C\left(\prod_{i=1}^{k-1} X_{i}, A\right)$ and $\prod_{i=1}^{k-1} X_{i}$ is a finite set with $n_{k}$ points. Thus there are $n_{k}$ ideals $\left\{I_{k, j}: 0 \leq j \leq\right.$ $\left.n_{k}-1\right\}$ in $A$ such that

$$
I_{k}=\left\{\sum_{j=0}^{n_{k}-1} a_{j} \delta_{\mathbf{j}}: a_{j} \in I_{k, j}\right\} .
$$

We have

$$
\sigma\left(\sum_{j=0}^{n_{k}-1} a_{j} \delta_{\mathbf{j}}\right)=\sum \alpha\left(a_{j}\right)\left(\delta_{\mathbf{j}} \circ \sigma_{0}^{-1}\right)=\sum_{j=0}^{n_{k}-2} \alpha\left(a_{j}\right) \delta_{\mathbf{j}+\mathbf{1}}+\alpha\left(a_{n_{k}-1}\right) \delta_{\mathbf{0}} .
$$


Hence the $\sigma$-invariance of $I_{k}$ implies that

$$
\alpha\left(I_{k, j}\right) \subseteq I_{k, j+1}
$$

for all $0 \leq j \leq n_{k}-2$ and

$$
\alpha\left(I_{k, n_{k}-1}\right) \subseteq I_{k, 0} .
$$

Thus, for all $j$, we have

$$
\alpha^{n_{k}}\left(I_{k, j}\right)=I_{k, j} .
$$

It follows from our assumptions that, for all $0 \leq j \leq n_{k}-1, I_{k, j}$ is either $A$ or $\{0\}$. In fact, the relations above show that either $I_{k, j}=A$ for all $j$ or $I_{k, j}=\{0\}$ for all $j$. It follows that $I_{k}$ is either $\mathscr{C}_{k}$ or $\{0\}$. Since $\left\{I_{k}\right\}$ is an increasing sequence whose union is dense in $I$, either $I=C(X, A)$ or $I=\{0\}$. This proves that $C(X, A)$ is $\sigma$-simple. In order to prove that the crossed product of $C(X, A)$ by $\sigma$ is simple it is left to show that the Connes' spectrum of $\sigma, \Gamma(\sigma)$, is the full unit circle. (See [9, Theorem 8.11.12]).

Let $\hat{A}$ and $\widehat{C(X, A)}$ be the set of equivalence classes of the irreducible representations of $A$ and $C(X, A)$ respectively. For every $x \in X$ and an irreducible representation $\tau$ of $A$ one can define an irreducible representation $\pi=\pi_{(x, \tau)}$ of $C(X, A)$ by $\pi(f)=\tau(f(x))$ and, conversely, every irreducible representation of $C(X, A)$ is equivalent to some $\pi_{(x, \tau)}$. Moreover $\tau_{1}$ and $\tau_{2}$ are equivalent if and only if the corresponding $\pi$ 's are equivalent (for the same $x$ ). Also, $\pi_{\left(x, \tau_{1}\right)}$ and $\pi_{\left(y, \tau_{2}\right)}$ are inequivalent whenever $x \neq y$. Hence we can write

$$
\widehat{C(X, A)}=\left\{\pi_{(x, \tau)}: x \in X, \tau \in \hat{A}\right\} .
$$

The automorphism $\sigma$ induces a map on $\widehat{C(X, A)}$ which we also denote by $\sigma$ (and similarly one has a map $\alpha$ on $\hat{A}$ ). Then

$$
\sigma\left(\pi_{(x, \tau)}\right)=\pi_{\left(\sigma_{0}^{-1}(x), \tau \circ \alpha\right)} .
$$

Suppose now that, for some $n \in Z, \sigma^{n}\left(\pi_{(x, \tau)}\right)=\pi_{(x, \tau)}$. Then it follows that $\sigma_{0}^{n}(x)=x$. But this is possible only if $n=0$ (from the definition of $\sigma_{0}$ ) and, thus, $\sigma$ acts freely on $\widehat{C(X, A)}$. We now use Theorem 10.4 of [8] (the equivalence of (i) and (v) there) to conclude that $\Gamma(\sigma)=\mathrm{T}$, completing the proof.

Proposition 3.6. The algebras $\mathscr{B}_{\alpha}\left(\left\{n_{k}\right\}\right)$ and $\mathscr{B}_{\alpha^{-1}}\left(\left\{n_{k}\right\}\right)$ are isomorphic.

Proof. One can prove the proposition by constructing isomorphisms between $\mathscr{B}_{\alpha}\left(n_{k}\right)$ and $\mathscr{B}_{\alpha^{-1}}\left(n_{k}\right)$ that are intertwined by the connecting maps in the direct limit. We prefer here to use the crossed product presentation. So let 
$X=\prod\left\{0, \ldots, m_{k}-1\right\}$ (where $\left.m_{k}=n_{k+1} / n_{k}\right)$ and let $\sigma_{0}$ be the odometer map as above. Recall that we defined $\sigma: C(X, A) \rightarrow C(X, A)$ by $\sigma(f)(x)=$ $\alpha\left(f\left(\sigma_{0}^{-1}(x)\right)\right)$. Write $\sigma^{\prime}(f)(x)=\alpha^{-1}\left(f\left(\sigma_{0}^{-1}(x)\right)\right)$. Then

$$
\mathscr{B}_{\alpha}\left(\left\{n_{k}\right\}\right) \cong C(X, A) \times_{\sigma} \mathrm{Z}
$$

and

$$
\mathscr{B}_{\alpha^{-1}}\left(\left\{n_{k}\right\}\right) \cong C(X, A) \times_{\sigma^{\prime}} \mathrm{Z} .
$$

We shall write $U$ for the unitary operator that satisfies $U f U^{*}=\sigma(f)$ for $f \in C(X, A)$ and such that $C(X, A)$ and $U$ generate the crossed product. Similarly we shall write $V$ for the unitary operator that, together with $C(X, A)$, generates the other crossed product and satisfies

$$
V f V^{*}=\sigma^{\prime}(f), \quad f \in C(X, A) .
$$

Now define a map $g$ on $X$ by

$$
g\left(x_{1}, x_{2}, \ldots\right)=\left(m_{1}-1-x_{1}, m_{2}-1-x_{2}, \ldots\right) .
$$

Then $g$ is a homeomorphism of $X$. It is also easy to check that

$$
g \circ \sigma_{0}=\sigma_{0}^{-1} \circ g \text {. }
$$

Let $\Psi$ be the map from $C(X, A) \times_{\sigma} \mathrm{Z}$ into $C(X, A) \times_{\sigma^{\prime}} \mathrm{Z}$ defined by setting

$$
\Psi(f)=f \circ g, \quad f \in C(X, A)
$$

and

$$
\Psi(U)=V^{*}
$$

We have, for $f$ in $C(X, A)$,

$$
\begin{aligned}
& V^{*} \Psi(f) V(x)=\sigma^{-1}(f \circ g)(x)=\alpha\left(f \circ g\left(\sigma_{0}(x)\right)\right) \\
& \quad=\alpha\left(f\left(\sigma_{0}^{-1}(g(x))\right)\right)=\sigma(f) \circ g=\Psi(\sigma(f)) .
\end{aligned}
$$

Hence the map $\Psi$ is a well defined *-homomorphism on $C(X, A) \times_{\sigma} Z$ Z. Similarly, by replacing the roles of $\alpha$ and $\alpha^{-1}$, one can define a ${ }^{*}$-homomorphism that is the inverse of $\Psi$, completing the proof of the proposition.

\section{Example: $\mathscr{B}_{\theta}\left(\left\{n_{k}\right\}\right)$}

In this section we discuss the special case where $A=C(\mathrm{~T})$ (the continuous functions on the unit circle), $\theta$ is a fixed irrational number and $\alpha=\alpha_{\theta}$ is the irrational rotation by $\theta$. Identifying $T$ with $R / Z$ we can write

$$
\alpha_{\theta}(f)(t)=f(t-\theta) .
$$


The algebra $C(\mathrm{~T}) \times{ }_{\alpha} \mathrm{Z}$ will be written (as is costumary) $A_{\theta}$ and the resulting limit algebra, $\mathscr{B}_{\alpha}\left(\left\{n_{k}\right\}\right)$ will be denoted $\mathscr{B}_{\theta}\left(\left\{n_{k}\right\}\right)$. It follows immediately from the results of Section 3 that this algebra (for a given increasing sequence $\left\{n_{k}\right\}$ of positive integers each dividing the next one) is simple and has a unique trace (denoted $\tau$ ).

Given a sequence $\left\{n_{k}\right\}$ as above, we shall write $\delta\left(n_{k}\right)$ (or simply $\delta$ if the sequence is fixed) for the associated supernatural number and $Q\left(\delta\left(n_{k}\right)\right.$ ) (or $Q(\delta))$ for the group of all rational numbers that can be written as a quotient $m / n_{k}$ for some $m \in \mathbf{Z}$ and $k \geq 1$. It is known that this group depends only on the supernatural number of the sequence.

THEOREM 4.1. Let $\theta$ be an irrational number and $\left\{n_{k}\right\}$ be a sequence of positive integers with $n_{k}$ dividing $n_{k+1}$ and $n_{1}=1$. Write $\delta\left(n_{k}\right)$ for its supernatural number and $\mathrm{R}^{+}$for the set of all non negative real numbers. Then

(i) $K_{0}\left(\mathscr{B}_{\theta}\left(\left\{n_{k}\right\}\right)\right)$ is isomorphic, as an ordered group, to $\left(Q\left(\delta\left(n_{k}\right)\right)+\theta \mathbf{Z}\right.$, $\left.\left(Q\left(\delta\left(n_{k}\right)\right)+\theta \mathbf{Z}\right) \cap \mathbf{R}^{+}\right)$.

(ii) Let $\tau_{*}$ be the map on $K_{0}\left(\mathscr{B}_{\theta}\left(\left\{n_{k}\right\}\right)\right)$ induced by the unique trace. Then

$$
\tau_{*}\left(K_{0}\left(\mathscr{B}_{\theta}\left(\left\{n_{k}\right\}\right)\right)\right)=Q\left(\delta\left(n_{k}\right)\right)+\theta \mathbf{Z} .
$$

Proof. Since $\mathscr{B}\left(n_{k}\right)$ is the algebra of $n_{k} \times n_{k}$ matrices over the irrational rotation algebra $A_{n_{k} \theta}$, the ordered group $K_{0}\left(\mathscr{B}\left(n_{k}\right)\right)$ is order isomorphic to $\left(\mathbf{Z}+\theta \mathbf{Z},\left(\mathbf{Z}+n_{k} \theta \mathbf{Z}\right) \cap \mathbf{R}^{+}\right)$. Let $\tau_{0}$ be the tracial state on $C(\mathbf{T})$ that one gets by integrating with respect to the normalized Lebegue measure (and then the unique trace $\tau$ on $\mathscr{B}_{\theta}\left(\left\{n_{k}\right\}\right)$ is $\Phi_{1}\left(\tau_{0}\right)$ in the notation of Proposition 3.1) then we write (as in Proposition 3.1) $\tau_{k}=\tau_{0} \circ \mathscr{E}_{k}$ (where $\mathscr{E}_{k}$ is the conditional expectation from $A_{n_{k} \theta}$ to $\left.C(\mathrm{~T})\right)$ and $\tilde{\tau}_{k}$ for the induced trace on $\mathscr{B}\left(n_{k}\right)$. Let $j: A_{n_{k} \theta} \rightarrow \mathscr{B}\left(n_{k}\right)=M_{n_{k}}\left(A_{n_{k} \theta}\right)$ be the map $j(X)=X e_{0,0}$. Then $j_{*}$ is an isomorphism of the $K_{0}$ groups and, since $\tilde{\tau}_{k} \circ j=\left(1 / n_{k}\right) \tau$, we have

$$
\left(\tilde{\tau}_{k}\right)_{*} \circ j_{*}=\frac{1}{n_{k}}\left(\tau_{k}\right)_{*} .
$$

Recall that we denote by $u_{n_{k}}$ the unitary that, together with a copy of $C(\mathrm{~T})$ generates the crossed product $C(\mathrm{~T}) \times_{\alpha_{\theta n_{k}}} \mathrm{Z}=A_{\theta n_{k}}$. Thus $u_{n_{k}}$ can be identified with the operator of rotation by $\theta n_{k}$ on $L^{2}(\mathrm{~T})$.

It is known that $\left(\tau_{k}\right)_{*}$ is an order isomorphism from $K_{0}\left(A_{\theta n_{k}}\right)$ onto $\mathbf{Z}+\theta n_{k} \mathbf{Z}$ $\left([1,10.11 .6]\right.$ or $\left[11\right.$, Example 5.8]). It follows that $\left(\tilde{\tau}_{k}\right)_{*}$ is an isomorphism of $K_{0}\left(\mathscr{B}\left(n_{k}\right)\right)$ onto $\left(1 / n_{k}\right) Z+\theta$ Z. Write $\gamma_{k}$ for the map $\gamma_{n_{k}, n_{k+1}}$ and $\left(\gamma_{k}\right)_{*}$ for the map it induces on the $K_{0}$ groups. Hence

$$
\left(\gamma_{k}\right)_{*}: K_{0}\left(\mathscr{B}\left(n_{k}\right)\right) \rightarrow K_{0}\left(\mathscr{B}\left(n_{k+1}\right)\right) .
$$


We have $\tilde{\tau}_{k+1} \circ \gamma_{k}=\tilde{\tau}_{k}$ (Proposition 3.1) and, thus, $\left(\tilde{\tau}_{k+1}\right)_{*} \circ\left(\gamma_{k}\right)_{*}=\left(\tilde{\tau}_{k}\right)_{*}$ and the map

$$
\lim _{\rightarrow}\left(\tilde{\tau}_{k}\right)_{*}: \lim _{\rightarrow} K_{0}\left(\mathscr{B}\left(n_{k}\right)\right) \rightarrow \mathrm{R}
$$

is a well defined order isomorphism into R. In fact, since $\tau=\lim _{\rightarrow} \tilde{\tau}_{k}$ (Proposition 3.1), the map $\lim _{\rightarrow}\left(\tilde{\tau}_{k}\right)_{*}$ is $\tau_{*}$. We also know that $\lim _{\rightarrow} K_{0}\left(\mathscr{B}\left(n_{k}\right)\right)$ is isomorphic to $K_{0}\left(\mathscr{B}_{\theta}\left(\left\{n_{k}\right\}\right)\right)$ as ordered groups ([11, Theorem 6.3.2]). It follows that

$$
\tau_{*}: K_{0}\left(\mathscr{B}_{\theta}\left(\left\{n_{k}\right\}\right)\right) \rightarrow \mathrm{R}
$$

is an order isomorphism. Its image is

$$
\cup\left(\left(1 / n_{k}\right) \mathbf{Z}+\theta \mathbf{Z}\right)=Q\left(\delta\left(n_{k}\right)\right)+\theta \mathbf{Z}
$$

Corollary 4.2. We have $\mathscr{B}_{\theta_{1}}\left(\left\{n_{k}\right\}\right) \cong \mathscr{B}_{\theta_{2}}\left(\left\{m_{k}\right\}\right)$ if and only if $\delta\left(n_{k}\right)=$ $\delta\left(m_{k}\right)$ and either $\theta_{1}+\theta_{2}$ or $\theta_{1}-\theta_{2}$ lies in $Q\left(\delta\left(n_{k}\right)\right)\left(=Q\left(\delta\left(m_{k}\right)\right)\right)$.

Proof. Assume first that the condition on the supernatural numbers and the $\theta$ 's holds. It follows from Proposition 3.6 that we can assume that $\theta_{1}-\theta_{2}$ lies in $Q\left(\delta\left(n_{k}\right)\right.$ ) (replacing $\theta_{2}$ by $-\theta_{2}$ if necessary). Then, for some $k \geq 1$, $n_{k} \theta_{1}-n_{k} \theta_{2}$ lies in Z. In fact, $n_{l} \theta_{1}-n_{l} \theta_{2} \in \mathbf{Z}$ for every $l \geq k$. The algebras $A_{n_{l} \theta_{1}}$ and $A_{n_{l} \theta_{2}}$ are then isomorphic. In fact, if we write $\psi_{l}$ for this isomorphism and apply $\psi_{l}$ entrywise we get an isomorphism $\tilde{\psi}_{l}$ of $\mathscr{B}_{\theta_{1}}\left(n_{l}\right)$ onto $\mathscr{B}_{\theta_{2}}\left(n_{l}\right)$. These isomorphisms intertwines the connecting maps and we get an isomorphism of the limit algebras.

For the other direction, assume the two algebras are isomorphic. Write $\eta$ for the isomorphism $\eta: \mathscr{B}_{\theta_{1}}\left(\left\{n_{k}\right\}\right) \rightarrow \mathscr{B}_{\theta_{2}}\left(\left\{m_{k}\right\}\right)$. If $\tau$ is the unique tracial state on $\mathscr{B}_{\theta_{2}}\left(\left\{m_{k}\right\}\right)$ then $\tau \circ \eta$ is the unique tracial state on $\mathscr{B}_{\theta_{1}}\left(\left\{n_{k}\right\}\right)$. Using Proposition 4.1 we have

$$
\begin{aligned}
Q\left(\delta\left(n_{k}\right)\right)+\theta_{1} \mathrm{Z} & =(\tau \circ \eta)_{*}\left(K_{0}\left(\mathscr{B}_{\theta_{1}}\left(\left\{n_{k}\right\}\right)\right)\right)=\tau_{*}\left(\eta_{*}\left(K_{0}\left(\mathscr{B}_{\theta_{1}}\left(\left\{n_{k}\right\}\right)\right)\right)\right) \\
& =\tau_{*}\left(K_{0}\left(\mathscr{B}_{\theta_{2}}\left(\left\{m_{k}\right\}\right)\right)\right)=Q\left(\delta\left(m_{k}\right)\right)+\theta_{2} \mathrm{Z} .
\end{aligned}
$$

It follows that, for every $k \geq 1$, there are $l \geq 1$ and $p, c$ in $\mathrm{Z}$ such that $1 / n_{k}=p / m_{l}+c \theta_{2}$. Since $\theta_{2}$ is irrational, $c=0$ and $n_{k} \mid m_{l}$. This shows that $\delta\left(n_{k}\right)$ divides $\delta\left(m_{k}\right)$ and, by symmetry, they are equal. Hence

$$
Q\left(\delta\left(n_{k}\right)\right)+\theta_{1} \mathbf{Z}=Q\left(\delta\left(n_{k}\right)\right)+\theta_{2} \mathbf{Z} .
$$

Thus there is some $k \geq 1$ and integers $a, b, c, d$ such that $\theta_{1}=b / n_{k}+a \theta_{2}$ and $\theta_{2}=c / n_{k}+d \theta_{1}$. Combining these equalities we find that $a d=1$. Hence either $a=d=1$ (and then $\theta_{1}-\theta_{2} \in Q\left(\delta\left(n_{k}\right)\right)$ ) or $a=d=-1$ (and then $\left.\theta_{1}+\theta_{2} \in Q\left(\delta\left(n_{k}\right)\right)\right)$. 
One can also compute the $K_{1}$ group of the algebra $\mathscr{B}_{\theta}\left(\left\{n_{k}\right\}\right)$ using the continuity of $K_{1}$.

Proposition 4.3. For every $\theta$ and $\left\{n_{k}\right\}$ as above,

$$
K_{1}\left(\mathscr{B}_{\theta}\left(\left\{n_{k}\right\}\right)\right) \cong Q\left(\delta\left(n_{k}\right)\right) \oplus \mathbf{Z} .
$$

Proof. It is known that $K_{1}\left(A_{n_{k} \theta}\right) \cong \mathrm{Z} \oplus \mathrm{Z}$ for every $k \geq 1$ and the generators of the group are the class $\left[u_{n_{k}}\right]$ of the unitary $u_{n_{k}}$ and the class $[v]$ where $v$ is the function in $C(\mathrm{~T})$ defined by $v(z)=z$ ([3, Example VIII.5.2] and [1, 10.11.6]). Fix $k \geq 1$, write $m=n_{k+1} / n_{k}$ and $\gamma_{k}$ for the map $\gamma_{n_{k}, n_{k+1}}$. The $K_{1}$ group of $M_{n_{k}}\left(A_{n_{k} \theta}\right)$ is again $\mathbf{Z} \oplus \mathbf{Z}$ and the generators are the classes of $\tilde{u}_{k}:=u_{n_{k}} e_{0,0}+\sum_{j=1}^{n_{k}-1} e_{j, j}$ and $\tilde{v}_{k}:=v e_{0,0}+\sum_{j=1}^{n_{k}-1} e_{j, j}$. We compute

$$
\gamma_{k}\left(\tilde{u}_{k}\right)=u_{n_{k+1}} e_{n_{k}(m-1), 0}+\sum_{l=0}^{m-2} e_{l n_{k},(l+1) n_{k}}+\sum_{i} e_{i, i}
$$

and

$$
\gamma_{k}\left(\tilde{v}_{k}\right)=\sum_{l=0}^{m-1} \alpha_{l n_{k} \theta}(v) e_{l n_{k}, l n_{k}}+\sum_{i} e_{i, i}
$$

where the last sums in both equations run over all integers $0 \leq i \leq n_{k+1}-1$ that are not multiples of $n_{k}$. But then it follows easily that

$$
\left(\gamma_{k}\right)_{*}\left(\left[\tilde{u}_{k}\right]\right)=\left[\tilde{u}_{k+1}\right]
$$

and

$$
\left(\gamma_{k}\right)_{*}\left(\left[\tilde{v}_{k}\right]\right)=m\left[\tilde{v}_{k+1}\right] .
$$

Hence, viewing $\left(\gamma_{k}\right)_{*}$ as a map from $\mathbf{Z} \oplus \mathbf{Z}$ to $\mathbf{Z} \oplus \mathbf{Z}$, we have

$$
\left(\gamma_{k}\right)_{*}:(a, b) \mapsto\left(n_{k+1} / n_{k} a, b\right) .
$$

Using the continuity of $K_{1}$ ([11, Proposition 8.2.7]) we get the result.

We close with the observation that every matrix algebra over an algebra of this class is again an algebra of this class. The precise statement is presented in the following proposition.

Proposition 4.4. For every $p \in \mathbf{N}$,

$$
M_{p}\left(\mathscr{B}_{\theta}\left(\left\{n_{k}\right\}\right)\right) \cong \mathscr{B}_{\theta / p}\left(\left\{p n_{k}\right\}\right) .
$$

Hence, for $p>1$, the algebras $\mathscr{B}_{\theta}\left(\left\{n_{k}\right\}\right)$ and $M_{p}\left(\mathscr{B}_{\theta}\left(\left\{n_{k}\right\}\right)\right)$ are non isomorphic. 
Proof. Write

$$
\mathscr{B}_{\theta}\left(\left\{n_{k}\right\}\right)=\lim _{\rightarrow}\left(\mathscr{B}_{\theta}\left(n_{k}\right), \gamma_{k}\right)
$$

where $\gamma_{k}$ stands for $\gamma_{n_{k}, n_{k+1}}$. Then

$$
M_{p}\left(\mathscr{B}_{\theta}\left(\left\{n_{k}\right\}\right)\right)=\lim _{\rightarrow}\left(M_{p}\left(\mathscr{B}_{\theta}\left(n_{k}\right)\right),\left(\gamma_{k}\right)_{p}\right)=\lim _{\rightarrow}\left(M_{p}\left(M_{n_{k}}\left(A_{\theta n_{k}}\right)\right),\left(\gamma_{k}\right)_{p}\right)
$$

where $\left(\gamma_{k}\right)_{p}$ is defined by applying $\gamma_{k}$ entrywise. We also have,

$$
\mathscr{B}_{\theta / p}\left(\left\{p n_{k}\right\}\right)=\lim _{\rightarrow}\left(\mathscr{B}_{\theta / p}\left(p n_{k}\right), \gamma_{k}^{\prime}\right)=\lim _{\rightarrow}\left(M_{p n_{k}}\left(A_{(\theta / p) p n_{k}}\right), \gamma_{k}^{\prime}\right)
$$

where $\gamma_{k}^{\prime}$ is $\gamma_{p n_{k}, p n_{k+1}}$. An element of $M_{p}\left(M_{n_{k}}\left(A_{\theta n_{k}}\right)\right)$ can be written, in an obvious way, as an $p n_{k} \times p n_{k}$ matrix over $A_{\theta n_{k}}$. Now perform the canonical shuffle on the matrix by applying the permutation

$$
\begin{aligned}
t_{k}:\left(0, \ldots, p n_{k}-1\right) & \\
& \mapsto\left(0, p, 2 p, \ldots,\left(n_{k}-1\right) p, 1, p+1, \ldots, p\left(n_{k}-1\right)-1, p n_{k}-1\right)
\end{aligned}
$$

on the rows and columns of the matrix. This defines an isomorphism

$$
\psi_{k}: M_{p}\left(M_{n_{k}}\left(A_{\theta n_{k}}\right)\right) \rightarrow M_{p n_{k}}\left(A_{\theta n_{k}}\right)
$$

that satisfies

$$
\psi_{k+1} \circ\left(\gamma_{k}\right)_{p}=\gamma_{k}^{\prime} \circ \psi_{k}, \quad k \geq 1 .
$$

The map $\psi:=\lim \psi_{k}$ is the required isomorphism.

The last statement follows from Corollary 4.2 since $\theta-\theta / p$ is irrational.

\section{REFERENCES}

1. Blackadar, B., K-Theory for Operator Algebras, 2nd. ed., Math. Sci. Res. Inst. Publ. 5 (1998).

2. Bunce, J., and Deddens, J., A family of simple $C^{*}$-algebras related to weighted shift operators, J. Funct. Anal. 19 (1975), 13-24.

3. Davidson, K. R., $C^{*}$-algebras by Example, Fields Inst. Monogr. 6 (1996).

4. Kajiwara, T., Pinzari, C., and Watatani, Y., Ideal structure and simplicity of the $C^{*}$-algebras generated by Hilbert bimodules, J. Funct. Anal. 159 (1998), 295-322.

5. Kribs, D., Inductive limit algebras from periodic weighted shifts on Fock space, New York J. Math. 8 (2002), 1-15.

6. Muhly, P. S., and Solel, B., Tensor Algebras over $C^{*}$-correspondences (Representations, dilations and $C^{*}$-envelopes), J. Funct. Anal. 158 (1998), 389-457.

7. Muhly, P. S., and Solel, B., On the simplicity of some Cuntz-Pimsner algebras, Math. Scand. 83 (1998), 53-73.

8. Olesen, D., and Pedersen, G. K., Applications of the Connes spectrum to $C^{*}$-dynamical systems III, J. Funct. Anal. 45 (1982), 357-390.

9. Pedersen, G. K., $C^{*}$-algebras and their Automorphism Groups, London Math. Soc. Monogr. (N.S.), 1979. 
10. Pimsner, M., A class of $C^{*}$-algebras generalizing both Cuntz-Krieger algebras and crossed products by Z, in Free Probability Theory, D. Voiculescu (ed.), Fields Inst. Comm. 12 (1997), 189-212.

11. Rørdam, M., Larsen, F., and Lausten, N. J., An Introduction to K-theory for $C^{*}$-algebras, London Math. Soc. Stud. Texts 49 (2000).

12. Schweizer, J., Dilations of $C^{*}$-correspondences and the simplicity of Cuntz-Pimsner algebras, J. Funct. Anal. 180 (2001), 404-425.

DEPARTMENT OF MATHEMATICS

TECHNION

HAIFA 32000

ISRAEL

E-mail: mabaruch@techunix.technion.ac.il 